\title{
Secondary wind transport of radioactive materials after the Fukushima accident
}

\author{
M. Yamauchi \\ Swedish Institute of Space Physics, Box 812, S-98128 Kiruna, Sweden \\ (Received July 11, 2011; Revised November 11, 2011; Accepted January 17, 2012; Online published February 27, 2012)
}

\begin{abstract}
Data from the radiation monitoring network surrounding the Fukushima Dai-ichi Nuclear Power Plant (FNPP) revealed that the radiation levels generally decayed faster at a highly-contaminated area than at neighboring moderately-contaminated areas during the first month after the Fukushima nuclear accident in March, 2011. Two possible mechanisms are considered: secondary transport of radioactive dust by wind or rain, and nonuniform radionuclide ratio of contamination between radioiodine $\left({ }^{131} \mathrm{I}\right)$ and radiocesium $\left({ }^{134} \mathrm{Cs}\right.$ and $\left.{ }^{137} \mathrm{Cs}\right)$. The composition data from soil does not favor the latter scenario, except for the local coastal region south of the FNPP, while inter-regional transport from the highly-contaminated area to the moderately-contaminated areas explains both the general difference in the decay rate in the entire area and the relatively slow decay at a high-dose rate anomaly $40 \mathrm{~km}$ northwest of the FNPP.
\end{abstract}

Key words: Radioactive contamination, iodine-cesium ratio, internal dose, Fukushima nuclear accident, resuspension of radioactive material, dust transport.

\section{Introduction}

The accident at the Fukushima Dai-ichi Nuclear Power Plant (FNPP) on 11 March, 2011, caused several releases of massive amounts of radioactive materials (NISA, 2011), resulting in substantial radioactive contamination in an area several hundred kilometers in diameter. A map of radioactive contamination levels has been produced after a coordinated large-manpower effort of soil sampling (MEXT, 2011). However, the contamination processes are not well understood. For example, large radioactive releases from the FNPP facility (venting and explosion) ended on 14 March, but large southbound radioactive plumes were observed on 20 March (Yamauchi et al., 2012, hereafter referred to as Paper 1). This indicates either sporadic secondary transport of radioactive materials that once contaminated the region close to the FNPP, or the release of new radioactive materials from the FNPP facility after 15 March. No estimate by measurements has been made on the radioactive outflux from the FNPP site because all monitoring stations within $20 \mathrm{~km}$ from the FNPP site were damaged by the 2011 Tohoku Earthquake that triggered the accident.

Understanding the secondary inter-regional transport of the radioactive materials is also important in other regions because the re-suspended radioactive material by wind may cause significant internal doses (IAEA, 2006). On this subject, Paper 1 and Takeda et al. (2011) used atmospheric electricity to diagnose different movements of radioactive dust until the end of May, 2011. This report compares the available radiation dose rate data to diagnose the inter-

Copy right(c) The Society of Geomagnetism and Earth, Planetary and Space Sciences (SGEPSS); The Seismological Society of Japan; The Volcanological Society of Japan; The Geodetic Society of Japan; The Japanese Society for Planetary Sciences; TERRAPUB.

doi:10.5047/eps.2012.01.002 regional dynamics of radionuclides.

\section{Data}

Figure 1 shows the radiation dose rate from 12 locations surrounding the FNPP on a logarithmic scale. These locations are shown in Fig. 2. After large increases of the radiation level on $20 \mathrm{March}$, when rain-induced fallout (wet contamination) to the ground took place (Paper 1), all locations show continuous decreases. The decay rate is not constant (not a straight line on the logarithmic scale), i.e., the time constant of the decay gradually increased in time. This is because long-lived radiocesium (half-life is about 2 years for ${ }^{134} \mathrm{Cs}$ and 30 years for ${ }^{137} \mathrm{Cs}$ ) contributed to the radiation dose rate after short-lived radioiodine ${ }^{131}$ I decayed quickly (half-life is about 8 days).

The decay rates are different at different places. For example, in Fig. 1, radiation dose rates at Iwaki, Kitaibaraki, and Takahagi showed a much faster decay compared to the other places during March, whereas the decay rates at these places became similar to those at the other places during May. Furthermore, some locations such as Iitate (blue) show different types of decay curves compared with the other locations. This paper discusses these differences. The rather regular daily variations in the decay curve seen in Fig. 1 are not discussed here.

Figure 3 shows the relative dose rates between pairs of locations. The three panels are for different reference sites (taken as the denominator for the ratio): MinamiSoma for the top panel, Iwaki for the middle panel, and Fukushimashi for the bottom panel. While all panels show rather constant ratios after 28 April, the ratios before 20 April changed at nearly all locations. Such changes indicate either secondary transport of the radionuclides (e.g., by wind, rain, or simple falling from trees) including subsurface migration 


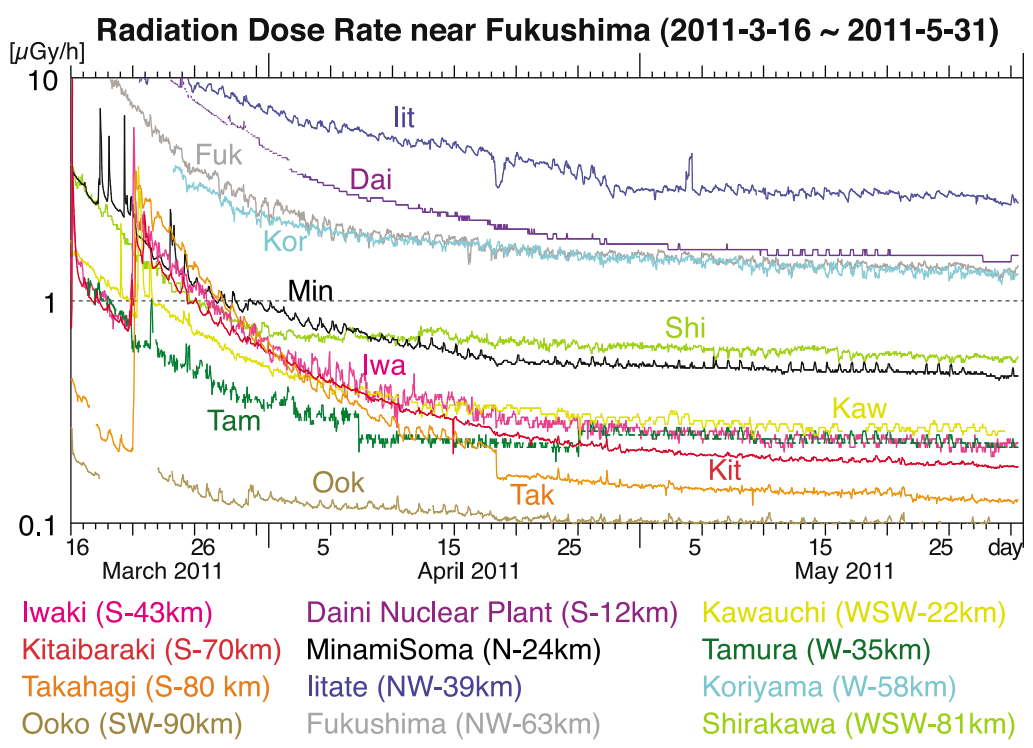

Fig. 1. One-hour resolution radiation dose rate data at 12 locations within $100 \mathrm{~km}$ from the Fukushima Dai-ichi Nuclear Power Plant (FNPP). The data is presented using a logarithmic scale over two and half months after the Fukushima nuclear accident (in UT) from 16 March although the accident started already on 11 March with the largest release on 14 March. Directions and distances from the FNPP (S: south, W: west, N: north) are given in the legend. The first three letters of each location are used as an abbreviation in the plot. The drop in values at Takahagi on 19 April 9 UT is due to a change of the measurement site. Measurements are made at $1 \sim 1.5 \mathrm{~m}$ height from the ground at all 12 locations using a NaI Scintillator with a supplement Ionization Chamber for $>10 \mu \mathrm{Gy} / \mathrm{h}$ cases.

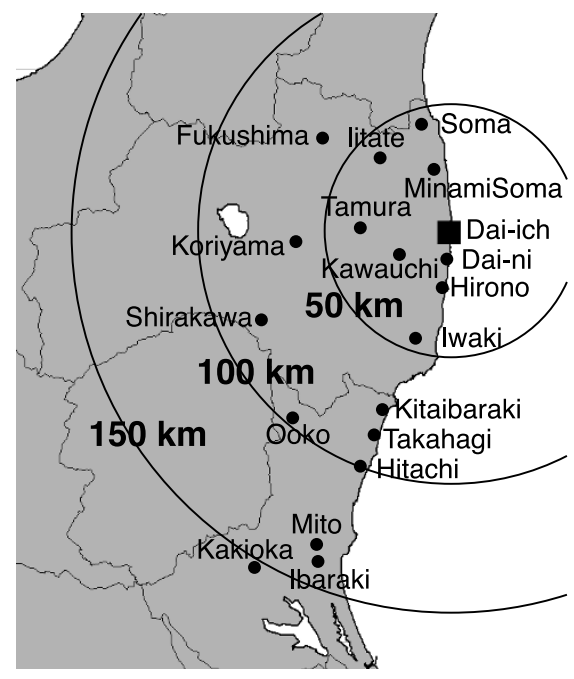

Fig. 2. The measurement locations that are given in Figs. 1 and 4. Fukushima Dai-ichi Nuclear Power Plant (Dai-ichi in the plot) and other places that are used in Paper 1 are also marked for reference.

(IAEA, 2006), or non-uniform radionuclide ratio (between ${ }^{131} \mathrm{I}$ and ${ }^{134,137} \mathrm{Cs}$ ) at different locations. One characteristic in Fig. 3 is that inter-regional ratios generally approached unity except in two regions (Iitate and Takahagi: both are mentioned later). Faster decay at a higher-contaminated area than at a lower-contaminated area has also been reported at a local-scale (10 km distance) near Mito and Ibaraki at $130 \mathrm{~km}$ south of the FNPP in Paper 1.

One possible reason for this general trend is redistribution from high-contamination areas to neighboring lowcontamination areas by wind, because the wind direction changed every few-to-tens hours according to the observation (not shown here). Paper 1 has drawn this conclusion using the atmospheric electric field data at Kakioka. Washout or subsurface migration by rain is less likely to be the reason, because the smooth approach to unity continued during 1-7 April when no rain was observed in the entire region. Non-correlation with the weather data also contradicts the scenario of radionuclides falling from trees. Inhomogeneity in the radionuclide ratio (the radiation dose rate should decay faster at higher I/Cs sites than at lower I/Cs sites) is the last candidate, but this is also unlikely the explanation for the general trend (as explained below).

Figure 4 shows the radionuclide ratio between ${ }^{131} \mathrm{I}$ and summation of ${ }^{134} \mathrm{Cs}$ and ${ }^{137} \mathrm{Cs}$ in the soil. The radioactive contamination in the soil was extensively examined by MEXT, Japan. In Fig. 4, different regions (cf. Fig. 2) are marked by different colors, and different sampling points are marked by different symbols. The figure demonstrates that the I/Cs ratios are similar at different sites in the same region, and that the decay is exponential (a straight line in the logarithmic-scaled plot) with a single decay constant (half-decay time of about 8 days). Since the half-life of ${ }^{131} \mathrm{I}$ is much shorter than those of ${ }^{134} \mathrm{Cs}$ and ${ }^{137} \mathrm{Cs}$, the I/Cs ratio in the soil is expected to decrease with about the half-life of ${ }^{131}$ I (about 8 days), as observed. From Fig. 4, one can extrapolate the I/Cs ratio on 21 March to be about 20, 8, 5, 3, and 2 in the regions south, southwest, west, northwest, and north, respectively, of the FNPP at distances of 30-40 km.

If the decay of the radiation dose rate is solely due to radioactive decay of ${ }^{131} \mathrm{I}$ and ${ }^{134,137} \mathrm{Cs}$, the inter-regional ratio of the radiation dose rate should be proportional to

$$
\left[e^{(t / T)}+C_{1}\right] /\left[e^{(t / T)}+C_{2}\right]
$$

where $T$ is nearly 8 days (the decay of ${ }^{134,137} \mathrm{Cs}$ is much 
Relative Radiation Dose Rate (March-May 2011) against MinamiSoma (N-24 km), Iwaki (S-43km), and Fukushima-shi (NW-63km)
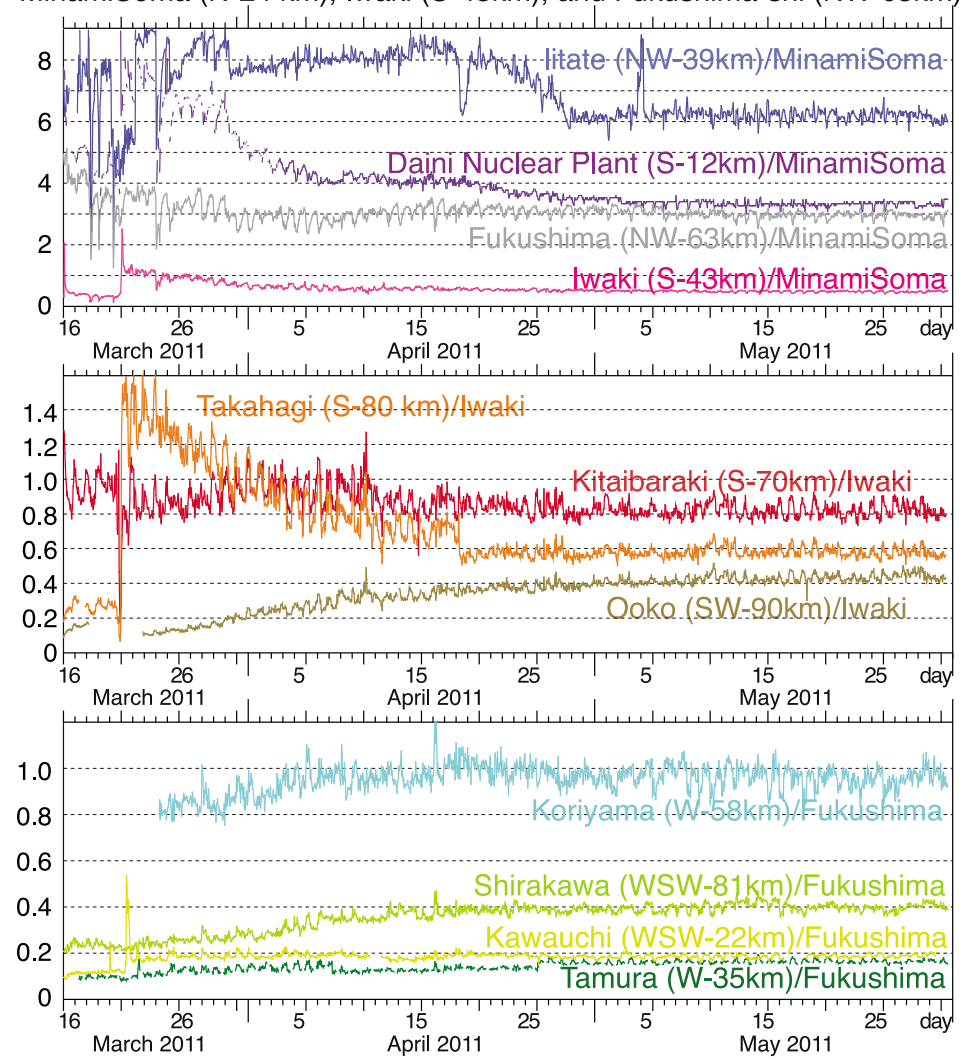

Fig. 3. Normalized radiation dose rate by reference towns: MinamiSoma for the top panel, Iwaki for the middle panel, and Fukushima-shi for the bottom panel. The same colors as Fig. 1 are used.

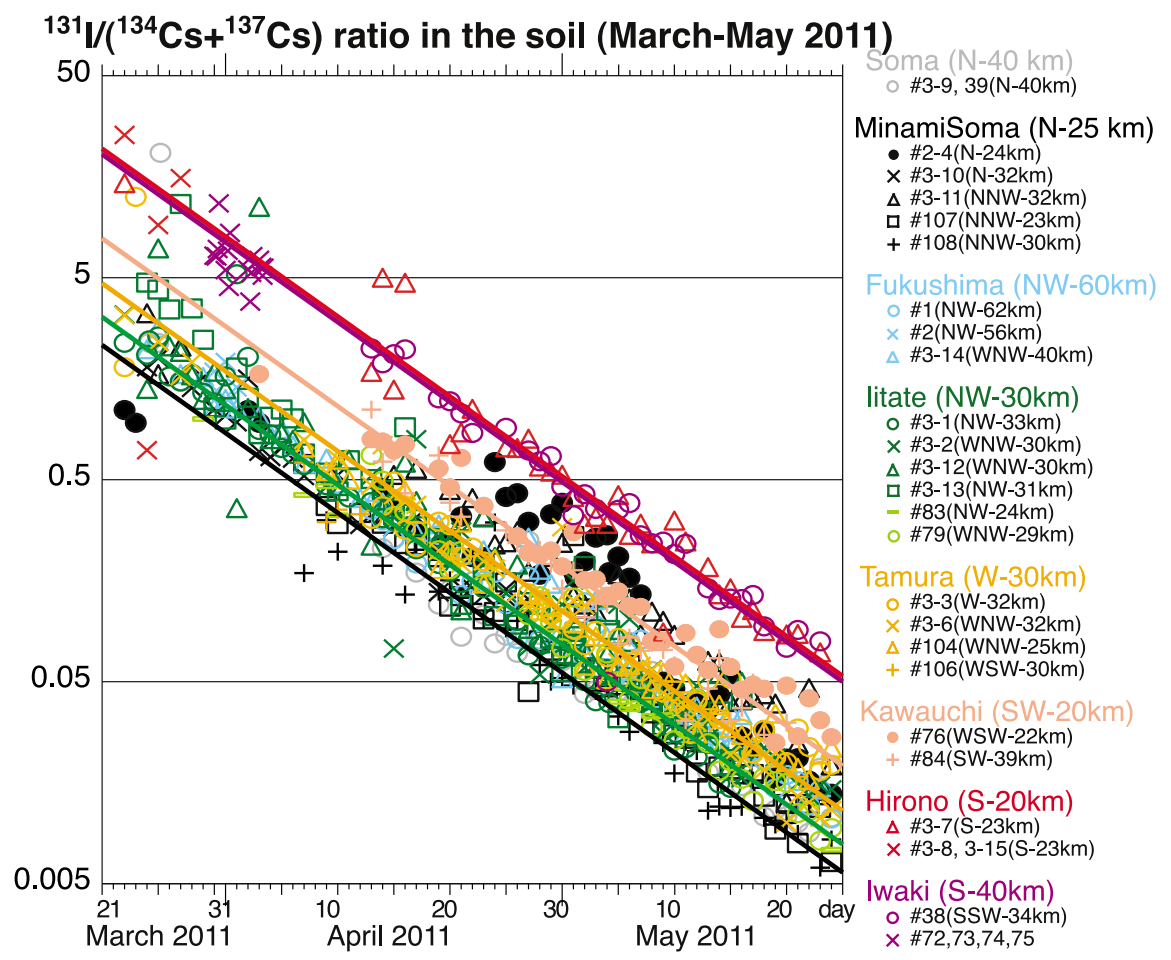

Fig. 4. Ratio of radioiodine $\left({ }^{131} \mathrm{I}\right)$ contamination to the radiocesium (summation of ${ }^{134} \mathrm{Cs}$ and ${ }^{137} \mathrm{Cs}$ ) contamination in soil at different locations in Fukushima prefecture. The code numbers of the sampling places are the same as the original data (contamination level in the soil) that is published by MEXT (MEXT, 2011). Directions and distances from the FNPP (S: south, W: west, N: north) are given in the legend. Approximate linear fitting is made region by region, except for anomaly points. 
smaller than the decay of ${ }^{131} \mathrm{I}$ ), and $C_{1}$ and $C_{2}$ are the I/Cs ratios at $t=0$ in the two regions, respectively. By using the I/Cs values estimated for 21 March $(t=0)$, one can make a quantitative prediction (not shown here), but this does not reproduce the general trend of all lines in Fig. 3. Therefore, the observed non-uniform I/Cs distribution is less likely the main reason.

The I/Cs ratio does not explain the unusual profile of the radiation ratio between Iitate and MinamiSoma in Fig. 3, either. Unlike the other plots in Fig. 3, the ratio slowly increased from 7.5 to 8.5 until 20 April before it quickly decreased during 21-27 April and stayed constant afterward. This anomaly comes mainly from the value at Iitate as shown in Fig. 1 (the radiation dose rate at MinamiSoma shows a similar profile to those at the other stations). The quick decrease during 21-27 April indicates an extra loss mechanism at Iitate other than the radioactive decay. The known mechanisms are redistribution by wind or rain; the latter is less likely because the rain data and Fig. 3 are not well correlated with each other (minor rain on 22, 23, 25 April at Iitate is much less than other rainfalls on 9, 11, 16, 19, 28 April).

The slower decay at Iitate than at the other locations before 20 April may also be due to the secondary transport, e.g., influx to Iitate or fallout from trees at Iitate. In secondary transport scenarios, quick decay can occur afterward by further transportation because new dry contamination is not as strongly attached to the surface as the old one (Paper 1). Note that Iitate is a hot region with high radioactive contamination compared with the other direction at the same distance from the FNPP (MEXT, 2011), but yet influx to Iitate is not impossible because the FNPP area is in the upwind direction from Iitate for the daily sea wind.

Let us examine the other unexpected profile of the radiation dose rate at Takahagi: it became higher than those at Iwaki or Kitaibaraki on $21 \mathrm{March}$, although Takahagi is located farther from the FNPP than these two places. Furthermore, the radiation dose rate decayed faster at Takahagi than at Iwaki or Kitaibaraki, and the value at Takahagi became smaller than the values at these two places already in the beginning of April. By contrast, the ratio between Iwaki and Kitaibaraki stayed relatively constant. These data are difficult to explain by the redistribution, while they can be explained if the I/Cs ratio at Takahagi was twice the value at Iwaki or Kitaibaraki, when the plume arrived at these three places on 20 March.

There is a link between Takahagi ( $80 \mathrm{~km}$ south of FNPP) and Daini Nuclear Plant (12 km south), and between Hirono (20 km south) and Iwaki (43 km south) for this plume: Daini Nuclear Plant shows a quick decay in Fig. 3 (corresponds to Takahagi) and Hirono shows as high a I/Cs ratio as Iwaki in Fig. 4. The ${ }^{131} \mathrm{I}$-rich part of radioactive plume most likely flew toward the ocean at around $10 \mathrm{~km}$ south of the FNPP, detouring over the ocean off from Iwaki and Kitaibaraki, returning toward the land at around $80 \mathrm{~km}$ south from the FNPP, while the part of the plume that flew over Hirono most likely arrived at Iwaki and Kitaibaraki.
The wind data at Iwaki and Kitaibaraki (not shown here) agrees with this interpretation.

\section{Summary and Discussion}

The decrease of the radiation dose rate after the nuclear accident at the FNPP is different at different locations for more than one month after the accident in March 2011. The uneven decrease is most likely due to secondary transport from high-contamination areas to moderate-contamination areas. Particularly, the area in the northwest (e.g., Iitate) most likely experienced a large outflux of radionuclides to the surrounding area during 21-27 April. Thus, some of the radioactive dust that once contaminated the surface was re-suspended and blown by wind, causing possible internal doses through air intake (IAEA, 2006) for more than one month after the accident. Iodine treatment could have been needed during this period. On the other hand, the uneven decay at coastal regions south of the FNPP (e.g., Takahagi) is most likely due to the non-uniform I/Cs ratio inside the radioactive plume on $20 \mathrm{March}$. The ${ }^{131} \mathrm{I}$-rich part of the plume flew toward the ocean at around $10 \mathrm{~km}$ south of the FNPP, returning toward the land at around $80 \mathrm{~km}$ south from the FNPP. Thus even the route of the plume can be inferred from the radiation dose rate data.

Acknowledgments. The data used here are published by the Ministry of Education, Culture, Sports (radioactivity.mext.go.jp/en/), $\quad$ Fukushima prefecture government (www.pref.fukushima.jp/ j/old\%5Fdata.html), Ibaraki prefecture government (www.pref.Ibaraki.jp/20110311eq/index2.html), and TEPCO site (www . tepco.co.jp/nu/fukushima-np/ $\mathrm{f} 2$ /index-j.html). These data, particularly the soil data, were taken by voluntary scientists who have made measurements in Fukushima regardless of the health risks. The meteorological data was provided by the Japan Meteorological Agency (www.jma.go.jp/jma/indexe.html). The author also thanks R. Ichimiya, H. Oda, I. Miyagi, A. Kondo, and M. Takeda for helpful suggestions.

\section{References}

IAEA, Environmental Consequences of the Chernobyl Accident and Their Remediation: Twenty Years of Experience, 166 pp, International Atomic Energy Agency IAEA, Vienna, 2006.

MEXT, Distribution Map of Radiation Dose around Fukushima Dai-ichi \& Dai-Ni NPP, press release by Ministry of Education, Culture, Sports, Science and Technology (MEXT), Japan, http: //www . mext.go. jp/english/incident/1305901.htm, 2011.

NISA, The Accident at TEPCO's Fukushima Nuclear Power Stations, Report of Japanese Government to the IAEA Ministerial Conference on Nuclear Safety, 7 June 2011, chapter VI, press release by Nuclear and Industrial Safety Agency (NISA), Japan, http: / / www . iaea . org/ newscenter/focus/fukushima/japan-report/, 2011.

Takeda, M., M. Yamauchi, M. Makino, and T. Owada, Initial effect of the Fukushima accident on atmospheric electricity, Geophys. Res. Lett, 38, L15811, doi:10.1029/2011GL048511, 2011.

Yamauchi, M., M. Takeda, M. Makino, T. Owada, and I. Miyagi, Settlement process of radioactive dust to the ground inferred by the atmospheric electric field measurement, Ann. Geophys., 30, 49-56, doi:10.5194/angeo-30-49-2012, 2012.

M. Yamauchi (e-mail: M.Yamauchi@irf.se) 